\title{
Bottom Detection Method of Side-Scan Sonar Image for AUV Missions
}

\author{
Huapeng Yu $\mathbb{D}^{1},{ }^{1}$ Ziyuan $\mathrm{Li}^{1,2}$ Dailin $\mathrm{Li}^{1}{ }^{3}$ and Tongsheng Shen $\mathbb{D}^{1}$ \\ ${ }^{1}$ National Innovation Institute of Defense Technology, Beijing, China \\ ${ }^{2}$ Science and Technology on Underwater Vehicle Laboratory, Harbin Engineering University, Harbin, China \\ ${ }^{3}$ College of Science, China University of Petroleum (East China), Qingdao, China \\ Correspondence should be addressed to Huapeng Yu; hpyu_qtxy@163.com and Tongsheng Shen; shents_bj@126.com
}

Received 18 September 2020; Accepted 6 October 2020; Published 17 October 2020

Academic Editor: Guang Li

Copyright (C) 2020 Huapeng Yu et al. This is an open access article distributed under the Creative Commons Attribution License, which permits unrestricted use, distribution, and reproduction in any medium, provided the original work is properly cited.

In order to obtain the measurement parameters of the sea bottom geomorphology or underwater objects, the first step in side-scan sonar (SSS) image processing is bottom detection. Due to the complexity of the marine environment, the acoustic signals received by SSS are usually polluted by noises, which affect its image quality and make the extraction of image features difficult. To address this problem, this study proposes an automatic detection method for the sea bottom line based on the actual experimental acquisition of SSS images, which is supposed to support the autonomous underwater vehicle (AUV) for intelligent target detection and classification. The proposed method comprises four main steps. First, the raw SSS data is analyzed to obtain a grayscale image, and the blind zone boundary of the image is obtained using the threshold method. Then, the noise characteristics of the image are analyzed and the denoising algorithm is optimized to effectively remove high-frequency noise. Next, spatial-temporal matching calculations are performed on each ping port and starboard data, and the accurate coordinates of first bottom returns are obtained through extreme value detection. Finally, automatic and accurate detection of the bottom line is realized according to the smooth processing of the coordinate sequence of first bottom returns. The experiments have demonstrated the effectiveness of the proposed method. As the method does not require human intervention in adjusting parameters during operation, the proposed method with a certain time window imposed during image acquisition will be suitable for AUV missions when the SSS is determined.

\section{Introduction}

Side-scan sonar (SSS) is an active sonar system, which mainly consists of a transducer array, peripheral auxiliary sensors, and a real-time data acquisition processing module. The transducer array is the core component of the SSS, which is usually designed to be receive-transmit combined line array [1]. SSS uses the principle of echo bathymetry to record scattered echo intensity from the sea bottom and generate sonar images according to echo intensities [1-3]. The SSS images can be utilized to observe changes in parameters such as sea bottom geomorphology, presence of obstructions, and types of sea bottom substrate, and its features have important applications in fields such as marine scientific research, marine engineering, and marine military.
In a SSS image, there is a clear junction line between the water column region and the seabed image region-called the sea bottom line, which is the distance from SSS to the sea bottom and an important parameter for measurements of the sea bottom topography or underwater objects, slant range correction, and SSS image grayscale equalization $[1,2,4,5]$.

Al-Rawi et al. [2] proposed two bottom detection methods: one to take the logarithm of the SSS echo data and use cubic spline regression algorithm for fitting and the other to filter the SSS data by sliding average filtering within a certain time window. Based on the data processed using these two methods, the center track line of the water column region is detected, and then, the first bottom return of each Ping data is obtained using the threshold method. Zhang 
et al. [6] solved the problem of the weak echoes of the sea bottom line in the actual SSS data by introducing the Laplacian of the Gaussian function, which is comparable to the bottom detection by commercial SSS data processing software. However, when the water noises are rather strong, the noises will be mistakenly extracted as the sea bottom line and it is difficult to obtain accurate bottom detection results, as the Gaussian smoothing filter can only eliminate the scales of intensity changes less than the smoothing factor. In view of the interferences of the SSS image in the water column region by the transmitting pulse, the sea surface echo, the wake, and the large area suspended matter, Zhao et al. [7, 8] proposed a last-peak detection method of the bottom line. Further, to tackle with ping data loss and ping observation anomaly that often exist in SSS measurements, Zhao proposed a Kalman filtering based sea bottom line repair method, which uses the asymptotic nature of the sea bottom variation and sea bottom line symmetry on SSS both sides. $\mathrm{Ku}$ et al. [9] measured single beam bathymetry and real-time depth of towed body using GPS time synchronization, and then, the bottom line was obtained by combination with these two kinds of data through interpolation and smoothing. Using actual measured data, the advantages of this method compared with the method proposed by Zhao et al. $[7,8]$ were verified. Moreover, the bathymetry measurement on this method was relatively less influenced by the environment and its accuracy was higher. According to the spatial characteristics of the continuous and dense distribution of the sea bottom line, Wang et al. [10] constructed a point set including the bottom points along the roughly parallel direction of the track and then clustered on the chain densely distributed along the track by the "densitybased spatial clustering of applications with noise (DBSCAN)" algorithm for chain searching to extract sea bottom line. Compared with the traditional bottom detection method, this method also has good stability and antiinterference ability in relatively complex environment. Yan et al. [11] used a one-dimensional convolution neural network to traverse and identify the backscatter intensity sequence in the SSS data and established a complete processing method for real-time sea bottom line detection and tracking, which has strong robustness in the field test environment.

An autonomous underwater vehicle (AUV) is capable of carrying detection equipment to accomplish automatic tasks such as marine surveys and seabed mapping [12-14]. Due to its advantages of high autonomy and low risk, AUVs are gradually being widely used, and their combinations with the SSS for underwater operations will become more frequent so that the superiority of SSS in target positioning and identification can be fully exploited $[2,12,14-16]$. However, few of the published research studies have mature engineering applications for the automatic bottom detection in SSS images suitable for AUV missions.

One of the main objectives of this study is to develop an automatic bottom detection method in SSS images suitable for AUV missions. The remainder of this paper is arranged as follows. Section 1 briefly introduces the characteristics of an SSS image and detailedly analyzes the published research studies and results of bottom detection. In Section 2, the current problems of bottom detection in SSS image for AUV missions are stated. Section 3 elaborates the proposed bottom detection method and verifies its effectiveness using the SSS actual test data on an AUV. Section 4 summarizes this study.

\section{Preliminaries and Problem Statements}

For AUV missions, there are two main deficiencies in the current research on SSS bottom detection.

(1) Under the hypothesis that there is either small and negligible or no blind zone between the port and starboard sonar transducer arrays, the installation of SSS is always taken as an ideal geometry. However, in actual working environments, the sonar transducers transmit sound waves to both sides, so there is a blind zone in the sonar image, and the space occupied by the blind zone in the sonar image should not be ignored. To avoid destroying the true geometry of the seabed image, it is necessary to consider eliminating the effects of the blind zone in the water column area.

(2) The published research results were obtained through complex postprocessing processes [7-9] or high online computing capability for AUV $[10,11]$. Moreover, due to the complex influence factors of SSS images, manual intervention is often required to set the initial value according to the situation, and most mature SSS image processing software, such as Triton and Prism, can only achieve reliable semiautomatic bottom detection $[7,10,12,17]$.

\section{Automatic SSS Bottom Detection}

3.1. Automatic Bottom Detection Procedure. Figure 1 shows the designed automatic bottom detection procedure for the SSS suitable for AUV missions. The procedure is described in four main steps.

\subsubsection{Step 1. Obtaining the Blind Zone Boundary according to} the SSS Parameters. In view of the geometry problem described in Section 2, first, it is necessary to calculate the parameters of the blind zone.

Since the blind zone is located directly below the SSS, the echo intensities of each ping are mutated from the blind zone to the outside, and the numerical values drop precipitously (see Figure 2). According to this feature, blind zone boundary detection can be realized. In general, the echo directly below the SSS is a strong positive emission wave, so a threshold can be used to detect the blind zone boundary detection.

Given a SSS, the ping data format is fixed (i.e., to 8, 16, or 32 bits). If the ping data bits of the SSS is $N$, then the threshold value for the blind zone boundary detection can be determined according to the measurement environment as follows: 


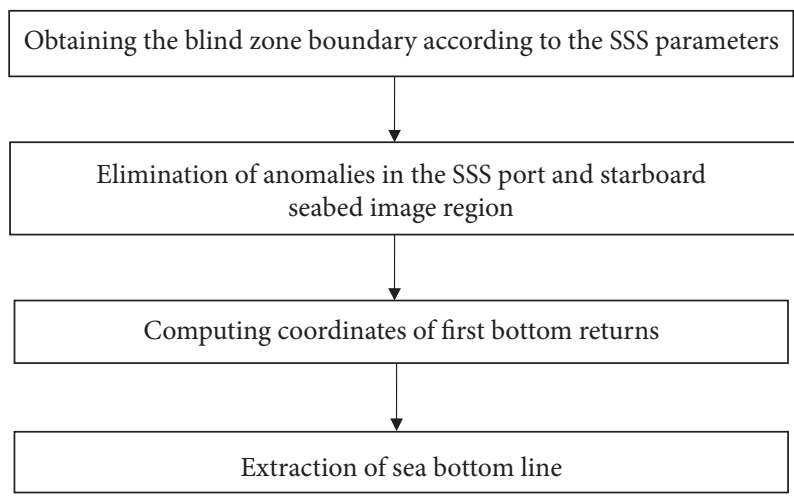

Figure 1: Automatic bottom detection procedure for SSS.

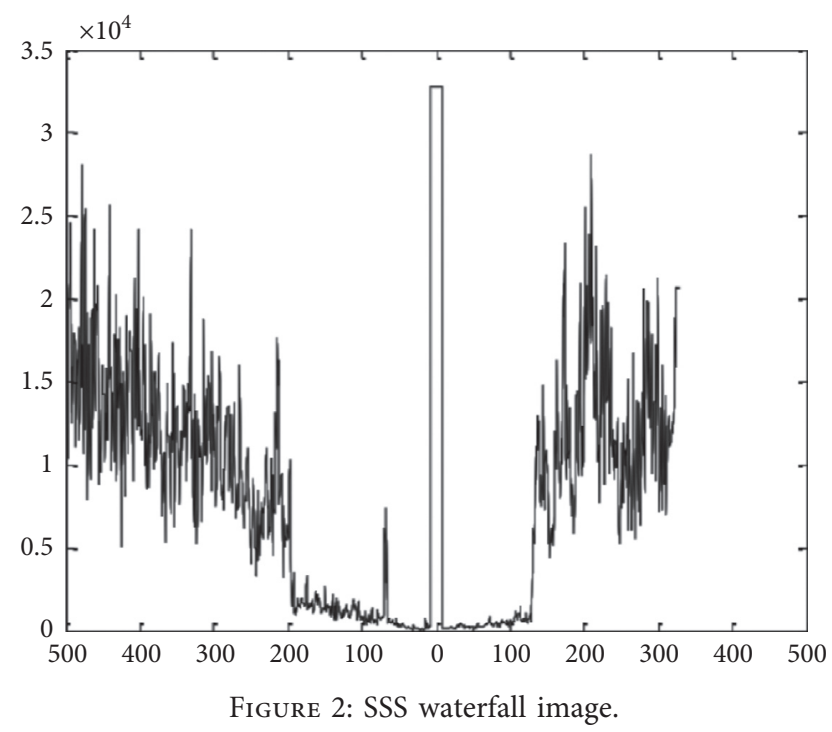

$$
D_{m}=2^{N-2}
$$

From the blind zone to the outside, the first echo intensities of each ping on the port and starboard sides that are less than the threshold value are denoted as $K_{\text {left }}$ and $K_{\text {right }}$, respectively. As the transducer arrays are mounted symmetrically on the port and starboard sides of the AUV with respect to its center axis, the width of the blind zone of each ping, $K_{m}$, according to the principle of bilateral symmetry, is determined as follows:

$$
K_{m}= \begin{cases}\frac{K_{\text {left }}+K_{\text {right }}-2}{2}, & \left(K_{\text {left }}+K_{\text {right }}\right) \text { is even, } \\ \frac{K_{\text {left }}+K_{\text {right }}-1}{2}, & \left(K_{\text {left }}+K_{\text {right }}\right) \text { is odd. }\end{cases}
$$

For each ping, the maximum sampling numbers of AUV left and right echo data are $K_{\text {leftmax }}$ and $K_{\text {rightmax }}$, respectively. Thus, the left echo data $\left[K_{m}, K_{\text {leftmax }}\right]$ and right echo data $\left[K_{m}, K_{\text {rightmax }}\right]$ of all pings, which imply the seabed image region, can be obtained.
3.1.2. Step 2. Elimination of Anomalies in the SSS Port and Starboard Seabed Image Region. The median filtering algorithm can be used to remove the anomalies in SSS echo data, that is, the high-frequency noise and outliers $[6,9]$. Herein, anomalies in both port and starboard SSS echo data are eliminated with an improved median filtering algorithm, and its calculation flow is as follows:

(1) Calculate the intensity median of every sampling echo in each ping of the port and starboard SSS data.

(2) Calculate the absolute intensity deviation of every sampling echo in each ping and determine the intensity value for the corresponding sampling echo based on its absolute intensity deviation. When the absolute intensity deviation is greater than a set value, the intensity median of the said sampling echo is taken as its new intensity value. Otherwise, no anomaly removal is performed and the intensity value for the said sampling echo is its sampling measured value. The above set value is a calculated value based on the echo intensity sampling values in each ping.

For example, for the SSS port echo data $\left[K_{m}, K_{\text {leftmax }}\right]$, let $[i, j]$ indicate the index of the $j$ th echo sampling measure of the $i$ th ping, and let $I_{i j}$ and $P_{\max }$ denote the intensity value of the $[i, j]$ th port echo data and the total number of SSS pings, respectively. Then, for all $[i, j]$ combinations satisfying $1<i<P_{\max }$ and $K_{m} \leq j<K_{\text {leftmax }}$, the following processing is performed:

(1) Calculate the intensity median $Z_{i j}$ using the $[i-1$, $j-1]$ th, $[i-1, j]$ th, $[i-1, j+1]$ th, $[i, j-1]$ th, $[i, j]$ th, $[i, j+1]$ th, $[i+1, j-1]$ th, $[i+1, j]$ th, and $[i+1, j+1]$ th sampling measured intensity values in port echo data, as well as the absolute intensity deviation $E_{i j}=\operatorname{abs}\left(I_{i j}-Z_{i j}\right)$.

It is obvious from the above example that the number of adjacent pings and the number of adjacent echo sampling measures in each ping are both determined according to the actual needs. The same can be said for the numerical calculation of the intensity value of every sampling echo in each ping of the starboard SSS data, which is described subsequently.

(2) Deciding absolute intensity deviation $E_{i j}$ [18]: if $E_{i j}>9.78+1.44 \times I_{i j}-8.86 \times 10^{-4} \times I_{i j}^{2}+2.95 \times 10^{-7} \times$ $I_{i j}^{3}-3.26 \times 10^{-11} \times I_{i j}^{4}$, then $Z_{i j}$ is used as the new intensity value for the $[i, j]$ th echo sampling measure in SSS port data; otherwise, no anomaly removal is performed.

For the SSS starboard echo data $\left[K_{m}, K_{\text {rightmax }}\right]$, let $[i, l]$ indicate the index of the $l$ th echo sampling measure of the $i$ th ping, and let $I_{i l}$ denote the intensity value of the $[i, j]$ th starboard echo data. Then, for all $[i, l]$ combinations satisfying $1<i<P_{\max }$ and $K_{m} \leq l<K_{\text {rightmax }}$, the following processing is performed: 
(1) Calculate the intensity median $Z_{i l}$ using the $[i-1$, $l-1] \mathrm{th},[i-1, l] \mathrm{th},[i-1, l+1] \mathrm{th},[i, l-1] \mathrm{th},[i, l] \mathrm{th}$, $[i, l+1]$ th, $[i+1, l-1]$ th, $[i+1, l]$ th, and $[i+1, l+1]$ th sampling measured intensity values in starboard echo data, as well as the absolute intensity deviation $E_{i l}=\operatorname{abs}\left(I_{i l}-Z_{i l}\right)$.

(2) Deciding absolute intensity deviation $E_{i l}$ : if $E_{i l}>9.78+1.44 \times I_{i l}-8.86 \times 10^{-4} \times I_{i j}^{2}$ $+2.95 \times 10^{-7} \times I_{i j}^{3}-3.26 \times 10^{-11} \times I_{i j}^{4}$, then $Z_{i l}$ is used as the new intensity value for the $[i, l]$ th echo sampling measure in SSS starboard data; otherwise, no anomaly removal is performed.

\subsubsection{Step 3: Computing Coordinates of First Bottom Returns.} According to the principle of bilateral symmetry of the sea bottom line, the first bottom returns received by the transducer arrays on the port and starboard sides of the SSS come from directly below AUV, the distances of the first bottom return echoes are equal, and the detected sea bottom lines on both sides are symmetrical to the AUV track [7, 9-11]. The above principle allows for better sea bottom line detection.

Herein, calculation of the coordinates of first bottom returns involves the following flow:

(1) The two spatial-temporal echo sampling measure sequences in each ping of SSS port and starboard data are matched and aligned.

The issue of unequal maximum numbers, $K_{\text {leftmax }}$ and $K_{\text {rightmax }}$, of the port and starboard echo sampling measures in one ping results in different temporal and spatial information implied by $I_{i j}$ and $I_{i l}$ when $l=j$. To maximize the usage of the principle of bilateral symmetry of the sea bottom line and to achieve a combined comparison between the port and starboard measures, the above problem must first be solved for removing as many influences of the suspended matter as possible.

In this study, bottom detection focuses on the water column region data and the mutated part of the seabed region data from the water column region. Therefore, to maintain the robustness of the bottom detection method, the SSS port and starboard echo data in each ping can be regarded as two spatialtemporal sequences for matching and alignment.
When performing matching the alignment, consistency in the spatial-temporal information of mutations from the water column region to the seabed region should be maintained. Thus, the dynamic time warping (DTW) algorithm is used to achieve this goal [19], which includes the following processes:

(a) For the $i$ th ping, there are two spatial-temporal sequences, the port echo sampling measures $\left\{I_{i j}\right.$, $\left.K_{m} \leq j \leq K_{\text {leftmax }}\right\}$, and the starboard echo sampling measures $\left\{I_{i l}, K_{m} \leq l \leq K_{\text {rightmax }}\right\}$. Take one echo from each of the two sequences and calculate the Euclidean distance $D\left(I_{i j}, I_{i l}\right)$ between them, where $K_{m} \leq j \leq K_{\text {leftmax }}, K_{m} \leq l \leq K_{\text {rightmax }}$. By calculating the Euclidean distances for all echoes in the two sequences, the Euclidean distance table can be constructed, as shown in Table 1.

(b) Search for the shortest path in the above Euclidean distance table. In the above table, dynamic planning is used to search for the shortest path from node $D\left(I_{i K m}, I_{i K m}\right)$ to node $D$ $\left(I_{i K \text { leftmax }}, I_{i \text { Krightmax }}\right)$. The shortest path must satisfy the condition that if the current node is $D$ $\left(I_{i j}, I_{i l}\right)$, then the next node must be selected among $D\left(I_{i j+1}, I_{i l}\right), D\left(I_{i j}, I_{i l+1}\right)$, and $D\left(I_{i j+1}, I_{i l+1}\right)$, and the chosen path must be the shortest.

(c) According to the nodes on the shortest path through the Euclidean distance table, two discrete sequences can be obtained as aligned echo sampling measure pairs with the same sequence length recorded as $K_{\max }$.

(2) Perform logarithm transformation on the matched and aligned port and starboard echo sampling measures in each ping; that is, take the base 10 logarithm.

(3) Calculate the standard deviation of the logarithmic sequence of the port and starboard echo sampling measures in each ping, and use them to construct a composite sequence of this ping.

Set a window to $W$, with a value as small as possible, to avoid flooding the mutant features of the first bottom return. Empirically, $W$ can be calculated as follows:

$$
W=\frac{\text { minimum height of SSS from seabed } \times \min \left(K_{\text {leftmax }}, K_{\text {rightmax }}\right)}{(3 \sim 6) \times \text { slant range }} .
$$

By calculating the standard deviation of the logarithmic sequence of the port and starboard echo sampling measures in each ping, two standard deviation sequences, denoted as $\left\{L_{i q}\right.$, $\left.1 \leq q \leq K_{\max }-W+1\right\}$ and $\left\{L_{i s}, 1 \leq s \leq K_{\max }-W+1\right\}$, are obtained, where the sequence length is $K_{\max }-W+1$.

In SSS echo data, there may be echo anomalies in the water column region, which will cause large fluctuations during the calculation of its standard 
TABLE 1: Euclidean distance table.

\begin{tabular}{lccc}
\hline$D\left(I_{i K m}, I_{i K m}\right)$ & $D\left(I_{i K m}, I_{i K m+1}\right)$ & $\ldots$ & $D\left(I_{i K m}, I_{i K \mathrm{Kightmax}}\right)$ \\
$D\left(I_{i K m+1}, I_{i K m}\right)$ & $D\left(I_{i K m+1}, I_{i K m+1}\right)$ & $\ldots$ & $D\left(I_{i K m+1}, I_{i K \mathrm{rightmax}}\right)$ \\
$\ldots$ & $\ldots$ & $\ldots$ & $\ldots$ \\
$D\left(I_{i K \text { leftmax }}, I_{i K m}\right)$ & $D\left(I_{i K \text { leftmax }}, I_{i K m+1}\right)$ & $\ldots$ & $D\left(I_{i K l \text { eftmax }}, I_{i K \mathrm{rightmax}}\right)$ \\
\hline
\end{tabular}

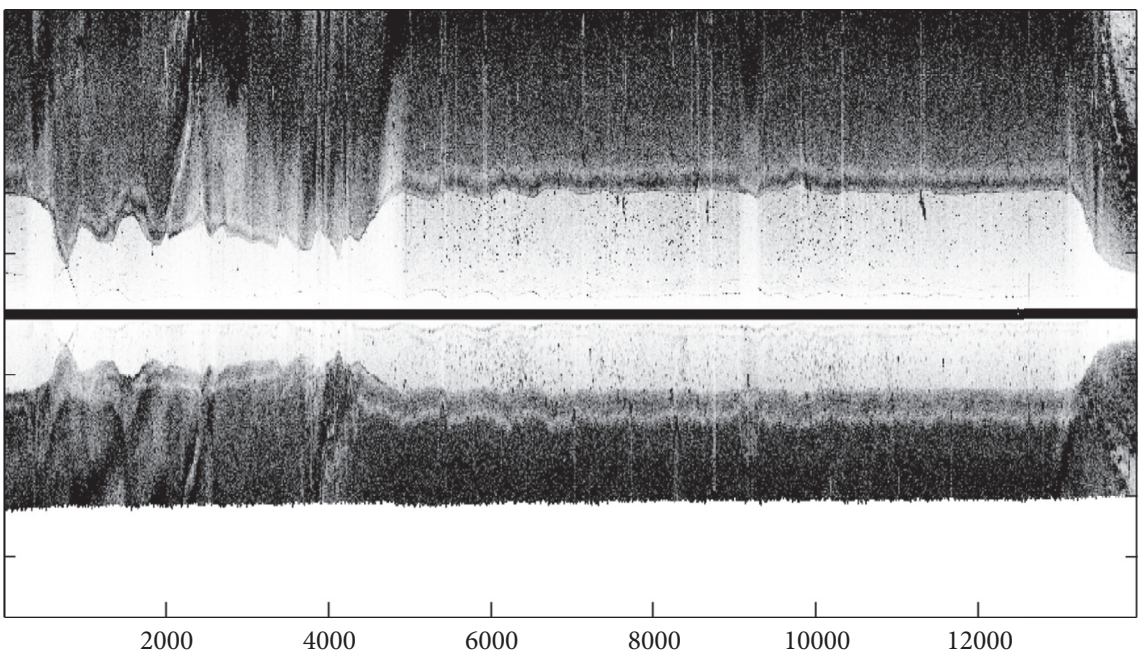

Figure 3: Echo data of a single ping scan line.

deviation sequence and, in turn, will affect the accuracy of subsequent extreme value detection. Therefore, to maximize the usage of the principle of bilateral symmetry of the sea bottom lines, we further take the minimum value of the port and starboard logarithmic sequence standard deviation of each ping. Consequently, for any $s=q$, $1 \leq q \leq K_{\max }-W+1$, and $1 \leq s \leq K_{\max }-W+1$, take $L_{i g}=\min \left(L_{i q}, L_{i s}\right)$, and a composite sequence of $i$ th ping $\left\{L_{i g}, 1 \leq g \leq K_{\max }-W+1\right\}$ is constructed.

(4) Perform extreme value detection on the composite sequence of each ping to obtain the extreme point coordinates.

Based on the characteristics of the SSS data, the sea bottom line comprises the first strong sea bottom echoes on the port and starboard side. Thus, the mutation of the echo intensity from the water column region to the seabed region will result in the first maximum value in the composite sequence $\left\{L_{i g}\right.$, $\left.1 \leq g \leq K_{\max }-W+1\right\}$. Therefore, the extreme point coordinates, denoted as $g_{b}$, can be obtained by performing extreme value detection.

(5) The coordinates of the port and starboard first bottom returns are obtained using the above extreme point coordinates.

The coordinates $q_{b}$ and $s_{b}$ of the standard deviation sequences corresponding to the logarithm of the obtained port and starboard echo sampling measures can be obtained using the extreme point coordinate $g_{b}$. In turn, the echo intensity $I_{b \text { left }}$ corresponding to the coordinate $q_{b}+W-1$ and $I_{b \text { right }}$ corresponding to the coordinate $s_{b}+W-1$ of the matched and aligned port and starboard sampling measure sequences are obtained by searching algorithms:

$\left\{I_{i j}, K_{m} \leq j \leq K_{\text {leftmax }}\right\}$, and the starboard echo sampling measures $\left\{I_{i l}, K_{m} \leq l \leq K_{\text {rightmax }}\right\}$.

For each ping, by searching the port sequence $\left\{I_{i j}\right.$, $\left.K_{m} \leq j \leq K_{\text {leftmax }}\right\}$ and finding the first echo intensity value that equals $I_{b \text { left }}$, the coordinate of the first bottom return is derived as $j_{b}$. Similarly, by searching the port sequence $\left\{I_{i l}\right.$, $\left.K_{m} \leq l \leq K_{\text {rightmax }}\right\}$ and finding the first echo intensity value that equals $I_{b r i g h t}$, the coordinate of the first bottom return is derived as $l_{b}$.

3.1.4. Step 4: Extraction of Sea Bottom Line. Considering the asymptotic nature of the sea bottom variation, precise sea bottom lines can be detected by sliding average filtering of the coordinates of the port first bottom returns $\left\{j_{b}\right\}$ and the coordinates of the starboard first bottom returns $\left\{l_{b}\right\}$ of all pings obtained through the above calculation [2].

3.2. Experimental Results and Discussion. In this experiment, the Sea Scan ${ }^{\circledR}$ ARC SCOUT Mk-II from the Marine Sonic Technology mounted on an AUV is used. The main operating parameters of the SSS are set to a ping frequency of $900 \mathrm{kHz}$ and a slant range of 10 meters. Raw data of all pings are collected through actual measurements in the offshore test.

The waterfall plot of the SSS echo data in reverse of the image displayed at 256 grayscale levels is shown in Figure 3. The corresponding separate SSS images for the port and 


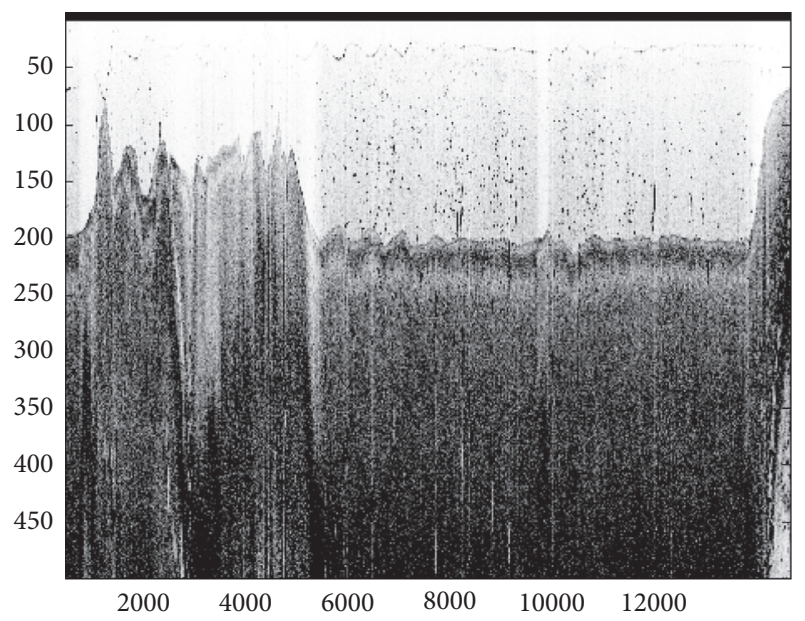

(a)

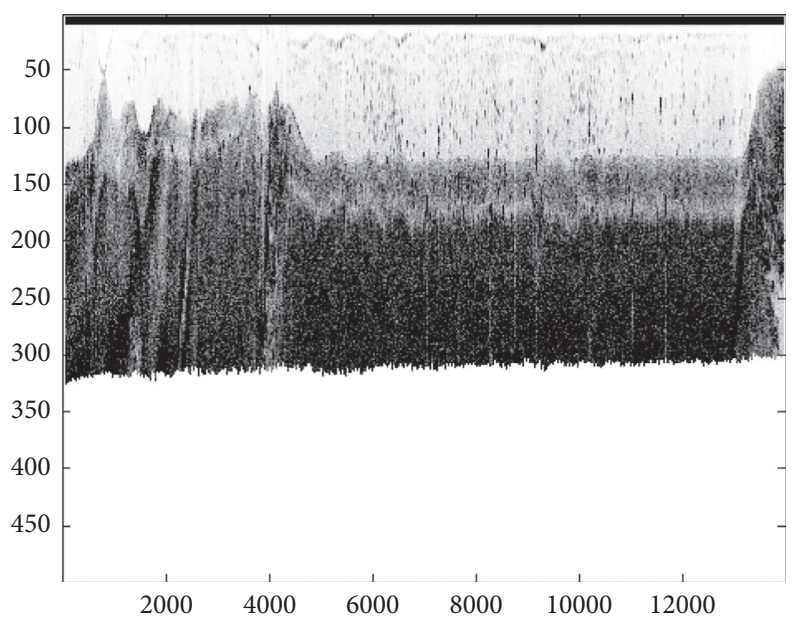

(b)

FIGURE 4: Separate SSS images for port and starboard sides with blind zone: (a) port side and (b) starboard side.

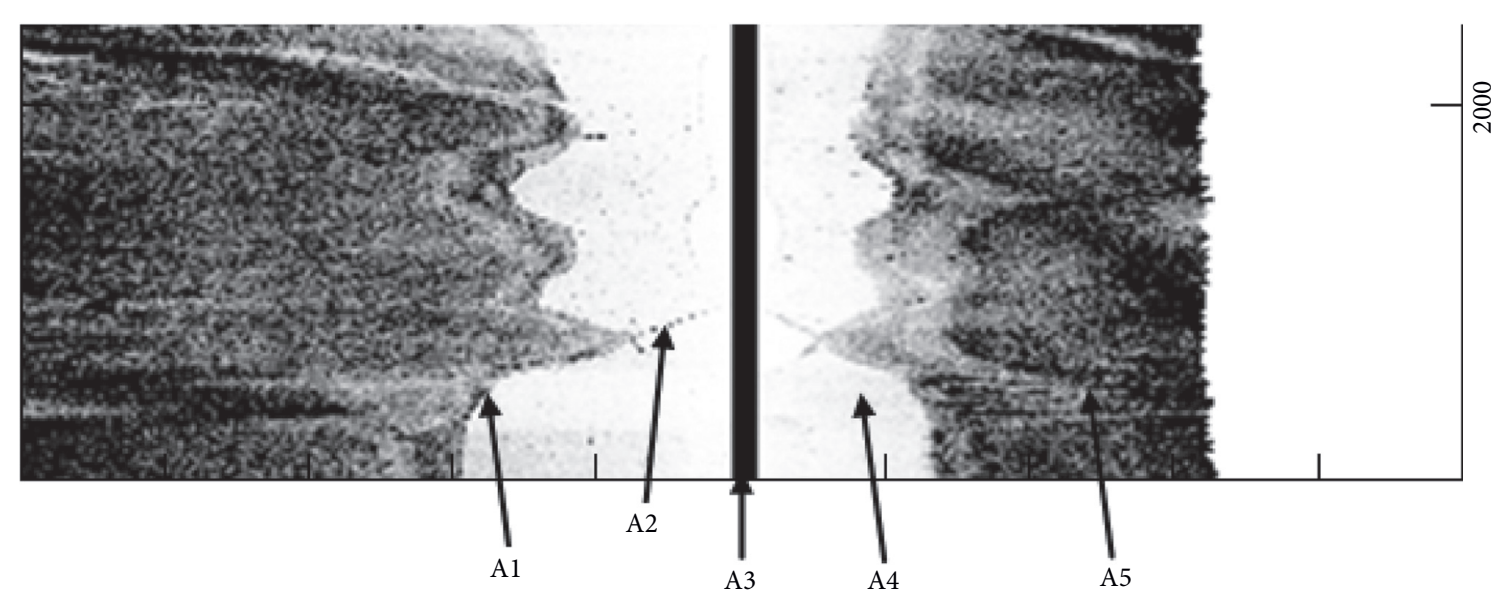

FIGURE 5: Main distribution parameters of the SSS waterfall image.

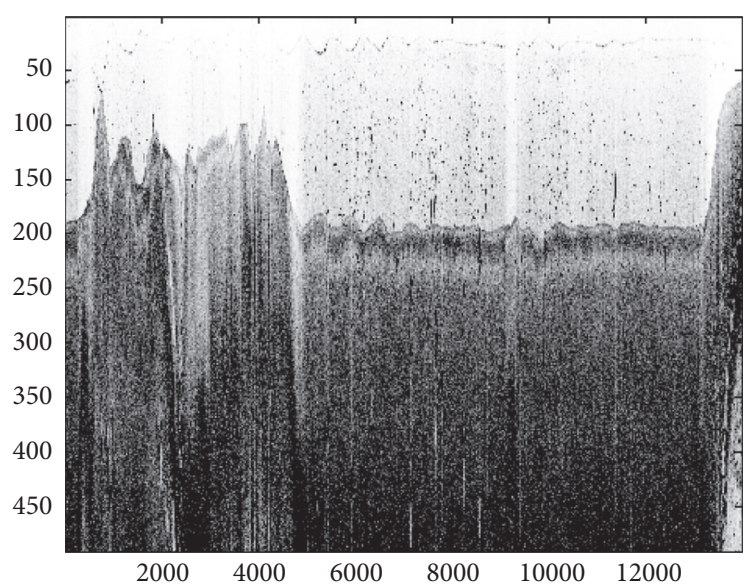

(a)

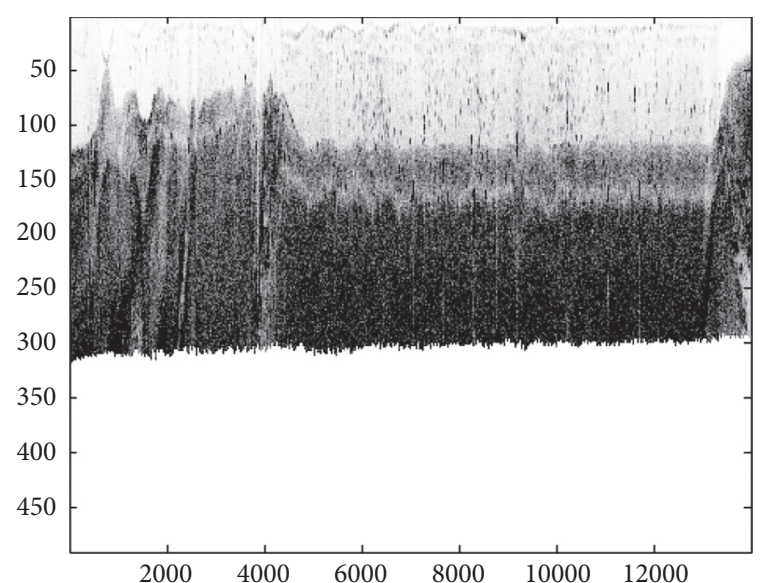

(b)

FIGURE 6: Separate SSS images of the port and starboard sides without blind zone: (a) port side and (b) starboard side. 


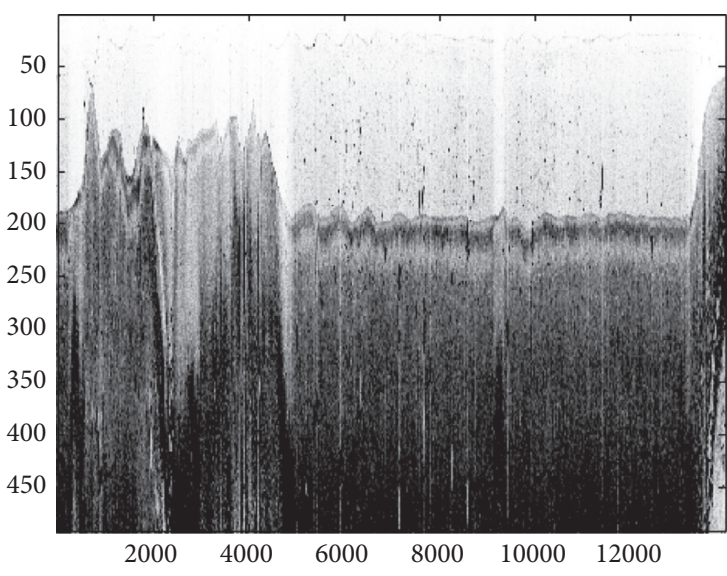

(a)

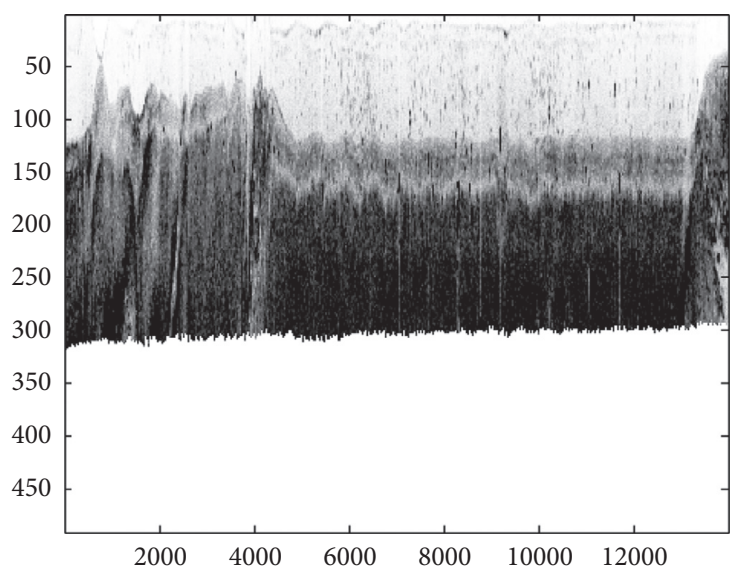

(b)

FIGURE 7: Separate SSS images of the port and starboard sides without blind zone after high-frequency noise and outliers filtering: (a) port side and (b) starboard side.

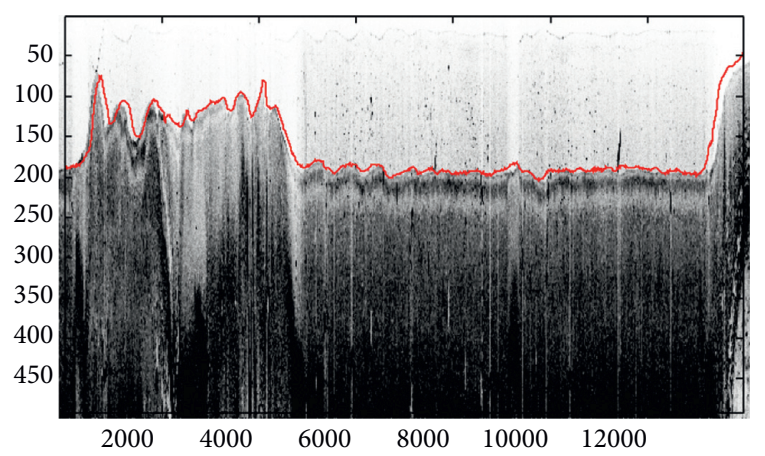

(a)

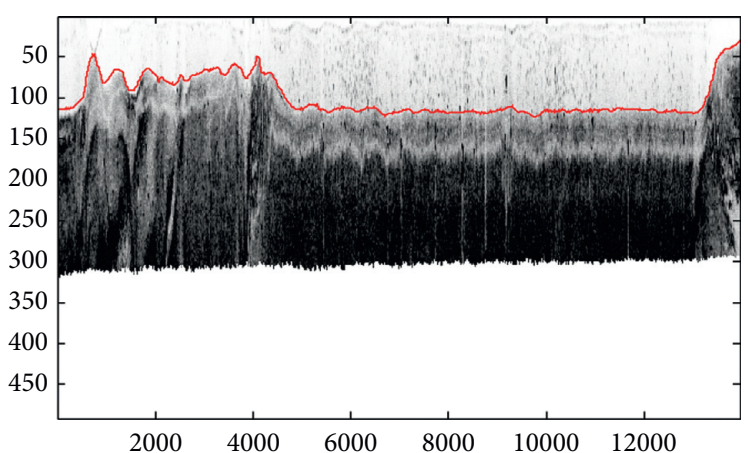

(b)

FIGURE 8: Detected sea bottom line marked separately on the port and starboard sides without showing the blind zone: (a) port side and (b) starboard side.

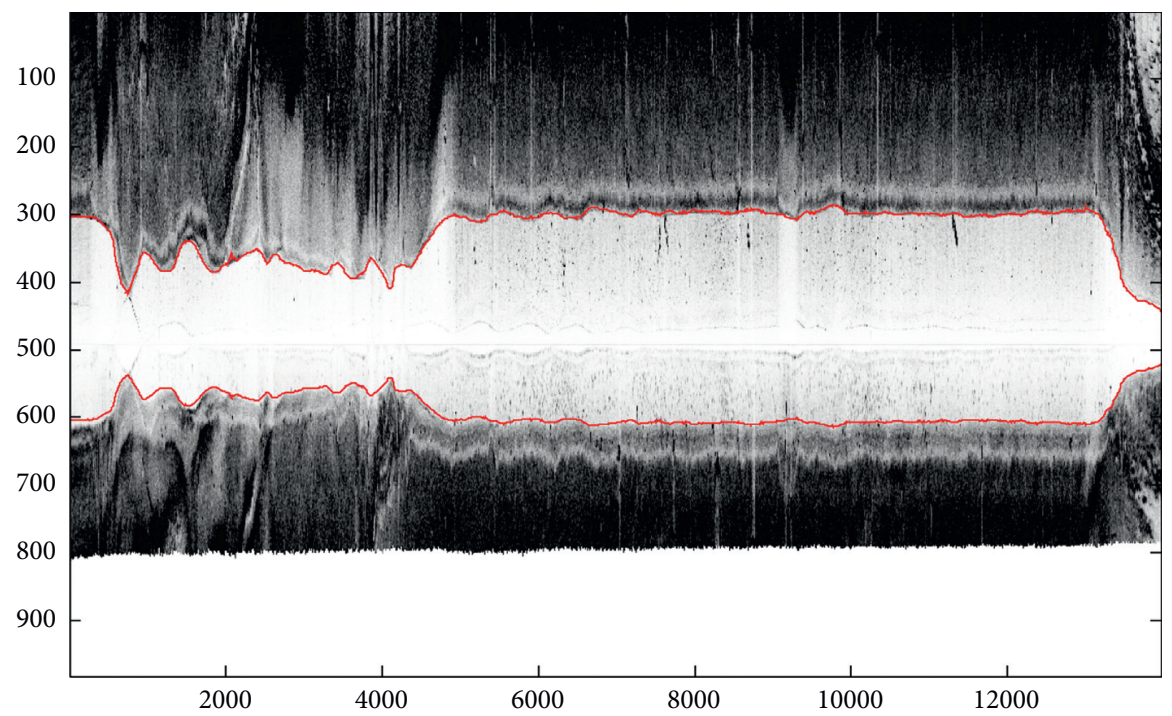

FIGURE 9: Sea bottom line marked in the waterfall of the SSS image without showing the blind zone. 
starboard sides with blind zone are shown in Figures 4(a) and $4(\mathrm{~b})$.

A waterfall plot of considerable echo data intercepted after the ping starting in Figure 3 is shown in Figure 5, where A1, A2, A3, A4, and A5 indicate the sea bottom line, sea surface line, blind zone, water column region, and seabed image region, respectively. It can be seen obviously that the darker the color, the stronger the echo intensity, and, conversely, the whiter (brighter) the color, the weaker the echo intensity.

Echo sampling measures of a single ping are shown in Figure 2. After removing the blind zone according to Step 1 in Section 3.1, separate SSS images of the port and starboard sides without showing the blind zone can be obtained, as shown in Figure 6.

In Figure 6, it can be seen that there are many anomalies in the echo data which may interfere with the detection of the sea bottom line and even cause false detection in complex environments, such as suspended matter present in the water column region. These echo anomalies are essentially high-frequency noise and outliers that can be effectively removed from the image during data processing according to an improved median filtering algorithm in Step 2 in Section 3.1, as shown in Figure 7.

The separate SSS images of the port and starboard sides without the blind zone after high-frequency noise and outliers filtering are shown in Figure 7. Compared to Figure 6 , the echo anomalies in the water column region can be eliminated to some extent.

Processed with Step 3 and Step 4 in Section 3.1, the detected sea bottom line marked separately on the port and starboard sides without showing the blind zone is shown in Figure 8, while the sea bottom line marked in the waterfall of the SSS image without showing the blind zone is shown in Figure 9.

\section{Conclusions}

In this study, the automatic bottom detection method of SSS image for AUV missions is presented. The existence of blind zone in the SSS image is taken into account, and the blind zone boundary of the SSS image is calculated based on the SSS parameters conveniently. The anomalies in SSS image are eliminated effectively using an improved median filtering algorithm. Further, to perform extreme value detection according to the principle of bilateral symmetry of the sea bottom lines, the SSS port and starboard echo data sequences in each ping are retained by using the logarithm of the space-time align-matched data. Therefore, an accurate sea bottom line is obtained through a sliding average filter with the coordinate sequences of the first bottom returns. The experimental results show that, without manual intervention to set the initial values online, the proposed method can overcome the strong noise interferences and achieve high detection accuracy and good detailed continuity in the process of sea bottom line detection.

\section{Data Availability}

The underlying data supporting the results of our study can be found at https://www.researchgate.net/profile/ Huapeng_Yu.

\section{Conflicts of Interest}

The authors declare no conflicts of interest.

\section{Acknowledgments}

This research was supported by the project of the National Natural Science Foundation of China (Grant no. 61803381).

\section{References}

[1] P. Blondel, The Handbook of Sidescan Sonar, Springer-Verlag, New York, NY, USA, 2009.

[2] M. Al-Rawi, F. Elmgren, M. Frasheri et al., "Algorithms for the detection of first bottom returns and objects in the water column in side-scan sonar images," in Proceedings of the A Vision for Our Marine Future Oceans '17, Aberdeen, UK, June 2017.

[3] C. Capus, A. C. Banks, E. Coiras, I. T. Ruiz, C. Smith, and Y. Petillot, "Data correction for visualisation and classification of sidescan sonar imagery," IET Radar Sonar and Navigation, vol. 2, no. 3, pp. 155-169, 2007.

[4] G. Guo, X. Wang, and H. Xu, "Review on underwater target detection, recognition and tracking based on sonar image," Control and Decision, vol. 2, no. 3, pp. 155-169, 2007.

[5] P. S. Chavez, J. Isbrecht, P. Galanis et al., "Processing, mosaicking and management of the Monterey Bay digital sidescan-sonar images," Marine Geology, vol. 181, no. 1-3, pp. 305-315, 2002.

[6] J. Zhang, G. Pan, and W. Ding, "Bottom track method in sidescan sonar data processing based on LOG operator," Marine Science Bulletin, vol. 29, no. 3, pp. 324-328, 2010.

[7] J. Zhao, X. Wang, and H. Zhang, "An automatic bottom extracting method for side-scan sonar image," Geomatics and Information Science of Wuhan University, vol. 42, no. 12, pp. 1797-1803, 2017.

[8] J. Zhao, X. Wang, H. Zhang, and A. Wang, "A comprehensive bottom-tracking method for sidescan sonar image influenced by complicated measuring environment," IEEE Journal of Oceanic Engineering, vol. 42, no. 3, pp. 619-631, 2017.

[9] A. Ku, X. Zhou, F. Wang, and Q. Tang, "A bottom extracting method for side-scan sonar image with single beam sounding," Marine Science Bulletin, vol. 38, no. 1, pp. 47-52, 2019.

[10] A. Wang, I. Church, J. Gou, and J. Zhao, "Sea bottom line tracking in side-scan sonar image through the combination of points density clustering and chains seeking," Journal of Marine Science and Technology, vol. 25, no. 3, pp. 1-17, 2019.

[11] J. Yan, J. Meng, and J. Zhao, "Real-time bottom tracking using side scan sonar data through one-dimensional convolutional neural networks," Remote Sensing, vol. 12, no. 1, pp. 1-21, 2019.

[12] P. Woock, "Side-scan sonar based SLAM for the deep sea," in Proceedings of the Joint Workshop of Fraunhofer IOSB and Institute for Anthropomatics, Vision and Fusion Laboratory 2010, pp. 63-74, La Bresse, France, July 2010.

[13] T. Glotzbach, M. Schneider, and P. Otto, "Cooperative line of sight target tracking for heterogeneous unmanned marine vehicle teams: from theory to practice," Robotics and $\mathrm{Au}$ tonomous Systems, vol. 67, pp. 53-60, 2015.

[14] J. Ferrand, J.-P. Malkasse, N. L. Bouffant, and F. Florin, "Automatic mine counter measure mission control for AUV systems," IFAC Proceedings Volumes, vol. 40, no. 17, pp. 7-12, 2007. 
[15] J. Petrich, M. F. Brown, J. L. Pentzer, and J. P. Sustersic, "Side scan sonar based self-localization for small autonomous underwater vehicles," Ocean Engineering, vol. 161, pp. 221226, 2018.

[16] D. Moreno, A. Burguera, and G. Oliver, "SSS-SLAM: an object oriented matlab framework for underwater SLAM using side scan sonar," in Proceedings of the Days Automatic, pp. 1-8, Valencia, Spain, September 2014.

[17] A. Wang, H. Zhang, X. Wang, and X. Shang, "Processing principles of side-scan sonar data for seamless mosaic image," Journal of Geomatics, vol. 42, no. 1, pp. 26-29, 2017.

[18] T. P. Bas, D. C. Mason, and N. C. Millard, "TOBI image processing-the state of the art," IEEE Journal of Oceanic Engineering, vol. 20, no. 1, pp. 85-93, 1995.

[19] D. J. Berndt and J. Clifford, "Using dynamic time warping to find patterns in time series," Knowledge Discovery and Data Mining, pp. 359-370, 1994. 